\title{
Re-sternotomy and coronary artery bypass grafting after omental flap procedure: a case report
}

\author{
Kayo Sugiyama ${ }^{1}$, Hirotaka Watanuki², Masaho Okada ${ }^{3}$, Yasuhiro Futamura ${ }^{4}$, Rintaro \\ Imazu $^{4}$, Satoshi Makino ${ }^{4}$, and Katsuhiko Matsuyama ${ }^{4}$ \\ ${ }^{1}$ Aichi Medical University \\ ${ }^{2}$ Aichi Ika Daigaku \\ ${ }^{3}$ Aichi Medical University School of Medicine \\ ${ }^{4}$ Aichi Medical University Hospital
}

July 25, 2020

\begin{abstract}
Few studies have reported re-sternotomy after an omental flap procedure. We describe the case of a 78-year-old man who received re-sternotomy after omental flap procedure for deep sternal wound infection and successfully underwent coronary artery bypass grafting. Although preoperative computed tomography showed funnel chest and limited space between the sternum and omentum, re-sternotomy was performed safely using circular electric sternum saw under partial cardiopulmonary bypass. Because the omentum functioned as cushioning material between the sternum and mediastinal organs, no injuries of the mediastinal organs occurred. An ultrasonic scalpel effectively dissected between the omentum and mediastinal organs, especially above the ascending aorta. The targeted coronary arteries were easily detected. The patient experienced no major cardiac or infectious events for three months. An ultrasonic scalpel is recommended for dissecting between the omentum and mediastinal organs.
\end{abstract}

\section{Introduction}

The omentum is an effective and potent tissue that can treat deep sternal wound infections because of its immunological properties ${ }^{1}$. Many studies reported the utility of an omental flap for severe mediastinitis after cardiac surgery ${ }^{2-4}$. Few reports described re-sternotomy after an omental flap procedure ${ }^{5}$, although there have been some similar reports ${ }^{6,7}$. Thick adhesions may be present after surgical treatment for mediastinitis $^{8}$, and detection of the targeted coronary arteries may be extremely difficult after surgical treatment for mediastinitis because of severe adhesion ${ }^{9,10}$. We report a 78-year-old-man who underwent re-sternotomy using CABG after omental flap for mediastinitis that was safely and successfully performed with a circular electric sternum saw.

\section{Case Report}

A 78-year-old man with severe coronary artery disease was referred to our hospital for coronary artery bypass grafting (CABG). He had undergone total arch replacement for an aortic arch aneurysm 9 years prior at another hospital. At that time, he underwent postoperative urgent debridement and an omental flap procedure for deep sternal wound infection during hospitalization. The split sternum was closed without wire banding. He recovered without recurrence of infection and was discharged.

Since then, he was treated for hypertension and diabetes mellitus at a local hospital. Three years prior, he received surgery for right-sided lung cancer. Six months prior, he underwent percutaneous catheter intervention due to severe triple-vessel disease. Follow-up coronary angiography revealed multiple in-stent 
restenoses despite strict medical treatment. Computed tomography (CT) showed funnel chest at the lower part of the sternum, with limited space between the sternum and omentum (Figure 1). There were also atheromatous changes in the descending aorta.

Considering his coexistent comorbidities, CABG with re-sternotomy was scheduled. Partial cardiopulmonary bypass was established through the right subclavian artery and right femoral vein for safe re-sternotomy by decompression of the cardiac volume. Re-sternotomy was safely performed using a circular electric sternum saw. The omentum functioned as a cushioning material between the sternum and mediastinal organs and appeared viable (Figure 2a); then, the left internal mammary artery was harvested. The space above the ascending aorta for proximal anastomosis, shown in Figure 2b, was dissected using an ultrasonic scalpel (Harmonic Scalpel ${ }^{\circledR}$; Ethicon Endo-Surgery, Cincinnati, OH, USA). The degree of adhesion in the pericardium was not severe; therefore, the targeted coronary arteries were easily detected. On-pump beating CABG was then performed using the left internal mammary artery to left anterior descending artery and saphenous vein grafts to obtuse marginal branch and distal site of the right coronary artery (Figure $2 \mathrm{~b}$ ). After proximal anastomosis of the coronary artery bypass at the native ascending aorta using a suture device, the viable omentum was reimplanted and covered the ascending aorta.

Weaning from cardiopulmonary bypass was uneventful, and the patient fully recovered without wound complications. Postoperative CT showed patency of all coronary artery bypass grafts and no remarkable infectious signs. The reimplanted omental flap was detected in the anterior mediastinum (Figure 3). The patient experienced no major adverse cardiac and infectious events for 3 months postoperatively.

The patient has provided permission to publish the features of his case. The identity of the patient has been protected.

\section{Discussion}

Re-sternotomy is more challenging than primary sternotomy because of adhesions ${ }^{8-10}$ and patients' poor health status. Increased reoperation risk through the reconstructed sternum is reported due to the small amount of tissue initially placed over the mediastinum ${ }^{8}$. Few reports described re-sternotomy after an omental flap procedure ${ }^{5}$, although there have been some similar reports ${ }^{6,7}$. In our case, severe adhesion between the omentum and mediastinal organs was expected because preoperative CT showed limited space between the sternum and omentum due to funnel chest. Re-sternotomy was completed safely because partial cardiopulmonary bypass was introduced by decompression of the cardiac volume and the omentum functioned as a cushioning material between the sternum and mediastinal organs.

Although no reports have described this effect, the omentum tissue can serve as a safety net for the mediastinal organs to prevent from injury. Adachi et al. reported re-sternotomy after omental flap in which dissection was safely performed ${ }^{5}$. Among 15 pediatric cardiac patients who underwent treatment for mediastinitis, 3 ( 2 with rectus flaps and 1 with pectoralis flap) underwent re-sternotomy ${ }^{6}$. The surgeries were uneventful because the rectus abdominus muscle was mobilized and removed ${ }^{6}$. According to Varennes et al, re-sternotomy after muscle flap repair could likely be accomplished without risk and heart transplantation could be carried out ${ }^{7}$. On the other hand, repeat median sternotomy after an omental flap procedure is more hazardous because of severe adhesions between the omentum and mediastinal structures ${ }^{11}$. Further studies regarding this feature of the omental flap are warranted.

Diversity of ultrasonic devices available may cause more efficient surgical procedures and improved patient outcomes $^{12}$. The ultrasonic scalpel is excellent in exfoliating adipose tissue. In our patient, an ultrasonic scalpel was useful for dissecting between the omentum and mediastinal organs, especially above the ascending aorta. Adachi et al. also reported the effectiveness of an ultrasonic scalpel for dissection during this procedure $^{5}$. Because Adachi's patient received ascending aortic and aortic valve replacement and mitral valve repair, the omental tissue was mobilized to the left side of the anterior mediastinum ${ }^{5}$, whereas in our patient, it was mobilized to the right side due to planned bypass grafting to the obtuse marginal branch.

Although it might be difficult to detect targeted coronary arteries after surgical treatment for mediastinitis 
because of severe adhesion ${ }^{9}, 10$, the coronary arteries were detected easily in our patient. According to Roselli et al, most injuries including heart chambers, great vessels and native coronary artery occurred on opening and during prebypass dissection ${ }^{10}$. In the present case, inflammation due to mediastinitis might have been limited to the area around the sternum and might not have extended into the pericardial cavity.

\section{Conclusion}

Our patient with funnel chest successfully underwent safe re-sternotomy and CABG after omental flap for mediastinitis. The omentum functioned as a cushioning material between the sternum and mediastinal organs. An ultrasonic scalpel was useful for dissecting between the omentum and mediastinal organs. Omental flap provides cushioning between the sternum and mediastinal organs. We recommend an ultrasonic scalpel for dissecting between the omentum and mediastinal organs.

\section{Acknowledgments}

We thank the Japan Medical Communication staff for reviewing and editing the manuscript. We also thank our colleagues for their helpful comments.

Conflict of interest None.

Authors' contributions: analysis: KS, KM; interpretation of data: KS; drafting and revising the paper: $\mathrm{KS}$, KM; final approval of the paper: all authors; agreement to be accountable for the integrity of the case reports: all authors. All authors have read and approved the final manuscript.

\section{References}

1. Spindler N, Etz CD, Misfeld M, Josten C, Mohr FW, Langer S. Omentum flap as a salvage procedure in deep sternal wound infection. Therapeutics and clinical risk management. 2017; 13: 1077-83.

2. Coselli JS, Crawford ES, Williams TW, Bradshaw MW, Wiemer DR, Harris RL, et al. Treatment of postoperative infection of ascending aorta and transverse aortic arch, including use of viable omentum and muscle flaps. Ann Thorac Surg. 1990; 50: 868-81.

3. Milano CA, Georgiade G, Muhlbaier LH, Smith PK, Wolfe WG. Comparison of omental and pectoralis flaps for poststernotomy mediastinitis. Ann Thorac Surg. 1999; 67: 377-81.

4. Zahra A, Sayed HF, Fouly MAE, Ghalwash M. Early management of deep sternal wound infections using omental flaps. Journal of the Egyptian society of cardio-thoracic surgery. 2018; 26: 141-5.

5. Adachi K, Yamaguchi A, Yuri K, Matsumoto H, Ito S, Kimura N, Adachi H. Valve replacement after omentum flap for mediastinitis; report of a case. Kyobu Geka. 2015; 68: 129-32.

6. Tortoriello TA, Friedman JD, McKenzie ED, Fraser CD, Feltes TF, Randall J, et al. Mediastinitis after pediatric cardiac surgery: A 15-year experience at a single institution. Ann Thorac Surg. 2003; 76: 1655-60.

7. Varennes BD, Tchervenkov CI, Kerrigan C, Dobell ARC. Sternotomy after muscle flap repair of sternal osteomyelitis and mediastinits. Journal of cardiac surgery. 1990; 5: 190-2.

8. Erez E, Katz M, Sharoni E, Katz Y, Leviav A, Vidne BA, et al. Pectoralis major muscle flap for deep sternal wound infection in neonates. Ann Thorac Surg. 2000; 69: 562-7.

9. Yaku H, Doi K. Redo coronary artery bypass grafting. Gen Thorac Cardiovasc Surg. 2014; 62: 453-60.

10. Roselli EE, Pettersson GB, Blackstone EH, Brizzio ME, Houghtaling PL, Hauck R, et al. Adverse events during reoperative cardiac surgery: Frequency, characterization, and rescue. J Thorac Cardiovasc Surg. 2008; 135: 316-23.

11. Heath BJ, Bagnato VJ. Poststernotomy mediastinitis treated by omental transfer without postoperative irrigation or drainage. J Thorac Cardiovas Surg. 1987; 94: 355-60. 
12. Welling AL, Scoggins PJ, Jeffrey JFC, Amaral JF. Superior dissecting capability of a new ultrasonic device improves efficiency and reduces adhesion formation. Glob Surg. 2017; 3: 1-5.

\section{Figure legends}

Figure 1. Preoperative CT shows funnel chest of the lower part of the sternum and limited space between the sternum and omentum (black dotted arrow).

Figure 2. (a) Intraoperative view shows viable omentum (black dotted arrow) above the ascending aorta. (b). Intraoperative view shows coronary artery bypass grafts (black arrowheads) anastomosed to the ascending aorta (black arrow) after omental flap mobilization (black dotted arrow) to the right side in the mediastinum.

Figure 3. Postoperative CT shows the reimplanted omental flap (white dotted arrow) in the anterior mediastinum.

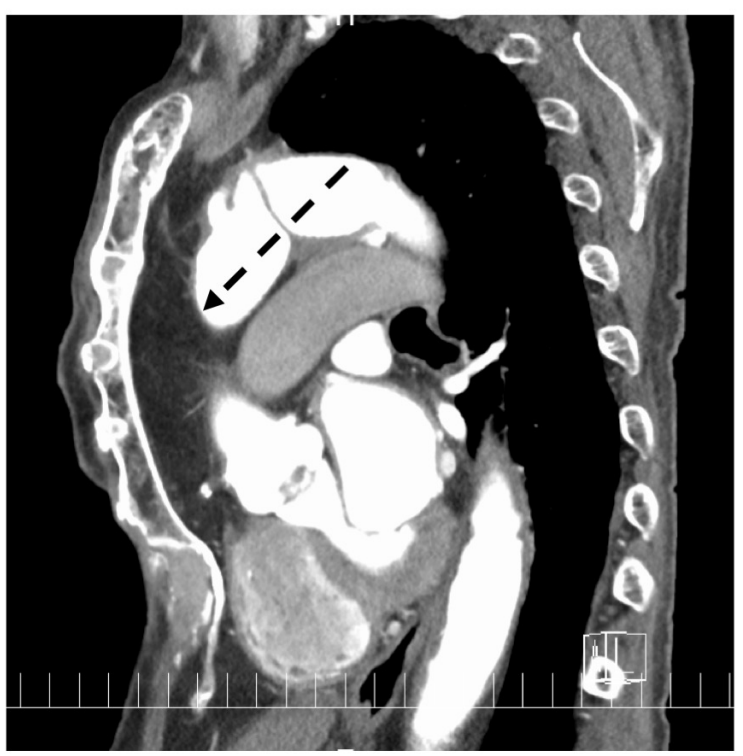



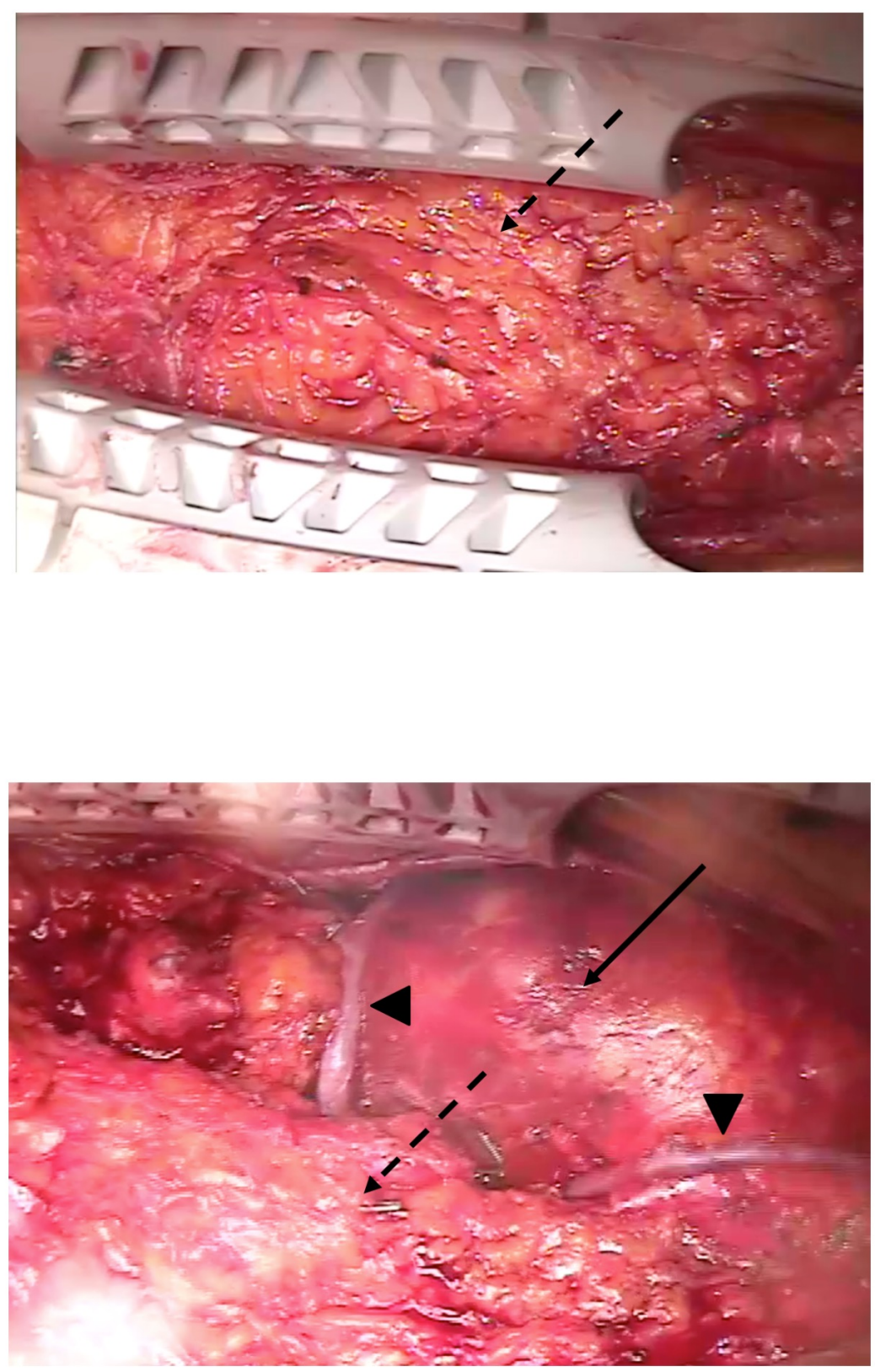


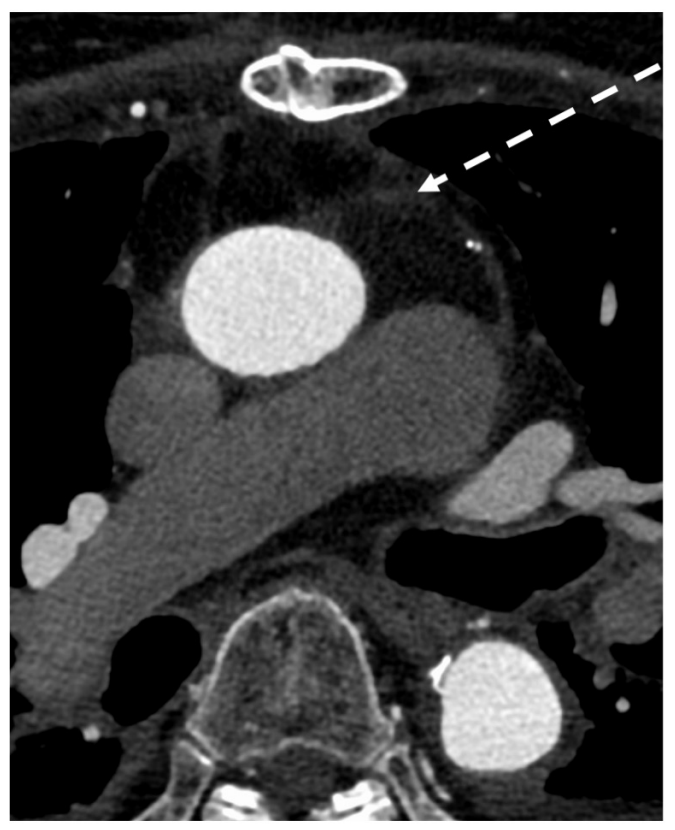

